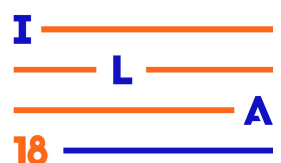

\title{
INTERAÇÃO EM DISPOSITIVOS TOUCHSCREEN PARA PESSOAS COM PARALISIA CEREBRAL: UMA REVISÃO BIBLIOGRÁFICA
}

\author{
INTERACTION IN TOUCHSCREEN DEVICES FOR PEOPLE WITH \\ CEREBRAL PARALYSIS: A BIBLIOGRAPHIC REVIEW
}

\author{
Carolina Savioli Marques Tavares ${ }^{1}$, Bel. \\ Murilo Scoz ${ }^{2}$, Dr.
}

(1) Universidade do Estado de Santa Catarina e-mail: carol.tavares.itu@gmail.com

(2) Universidade do Estado de Santa Catarina e-mail: muriloscoz@gmail.com

Paralisia cerebral, touchscreen, interação digital.

\begin{abstract}
Considerando a paralisia cerebral, esta pesquisa objetivou observar e identificar pesquisas atuais acerca do uso de dispositivos touchscreen como tecnologias assistivas voltadas às disfunções motoras finas de indivíduos com este acometimento através de uma revisão bibliográfica sistemática. Com os resultados obtidos, apresenta-se informações relevantes à usabilidade e inclusão deste público.
\end{abstract}

Cerebral palsy, touchscreen, digital interaction.

Considering cerebral palsy, this research aimed to observe current research regarding the use of touchscreen devices as assistive technology for fine motor dysfunctions of individuals with this impairment through a systematic bibliographical review. With the results obtained, relevant information towards usability and inclusion for this audience is presented.

\section{Introdução}

Ao considerarmos o avanço tecnológico e a crescente disseminação de dispositivos móveis com tela sensível ao toque à população, como smartphones e tablets, nota-se a importância de torná-los acessíveis à maior porção populacional possível, incluindo indivíduos com deficiência (visual, auditiva, motora, mental ou intelectual), que, segundo dados do Instituto Brasileiro de Geografia e Estatística (IBGE), realizado em 2010, compõem aproximadamente 45 milhões de pessoas ou $23.9 \%$ da população brasileira.

Entre estas condições, destaca-se a paralisia cerebral (PC), a mais frequente deficiência motora infantil, com estimativa de presença em 2 a cada 1.000 bebês nascidos vivos em países desenvolvidos e 7 a cada 1.000 em países subdesenvolvidos, como o Brasil (CIASCA; MOURA-RIBEIRO; TABAQUIM,
2006). A paralisia cerebral, segundo descrição da SCPE (Surveillance of Cerebral Palsy in Europe), designa um "conjunto de dificuldades de movimento que surgem quando as regiões do cérebro que controlam esses movimentos não funcionam devidamente". A paralisia cerebral descreve, assim, "um grupo de desordens permanentes do desenvolvimento do movimento e postura atribuído a um distúrbio não progressivo que ocorre durante $o$ desenvolvimento do cérebro fetal ou infantil, podendo contribuir para limitações no perfil de funcionalidade da pessoa" (BRASIL, 2014, p. 8). As lesões da paralisia cerebral são consistentes, não progressivas e podem afetar as funções motoras, cognitivas, a visão, a audição e ocasionar epilepsia. A paralisia cerebral "se manifesta na primeira infância, usualmente antes dos 18 meses de idade" (BRASIL, 2014, p. 19). 


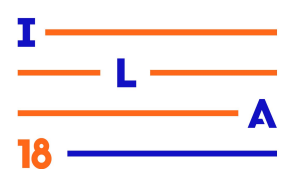

Entre os acometimentos possíveis, as disfunções motoras são as mais comuns, podendo limitar ou modificar a interação deste público com artefatos tecnológicos e interfaces digitais. Apesar da variabilidade entre casos da paralisia cerebral, estas disfunções conferem categorizações, como o Sistema de Classificação da Função Motora Grossa (GMFCS), com foco postural e nos membros inferiores, que "baseia-se no movimento iniciado voluntariamente, com ênfase no sentar, transferências e mobilidade" (BRASIL, p. 11, 2014), e Sistema de Classificação das Capacidades de Manipulação (MACS), que "descreve como as crianças com paralisia cerebral (PC) usam suas mãos para manipular objetos em atividades diárias" (ELIASSON et al, 2010), levando em conta o desempenho dos membros superiores.

As diversas interações cotidianas deste público e, mais especificamente, as digitais, se dão por meio de tecnologias assistivas (TA), que podem facilitar a comunicação, movimentação e educação. As TAs, especificamente para os casos de paralisia cerebral, consistem em "recursos e serviços que visam promover a funcionalidade e a autonomia da pessoa com paralisia cerebral, minimizando os problemas e as dificuldades decorrentes dessa condição de saúde" (BRASIL, p. 45, 2014).

Saffer (2009) ressalta que entramos em uma nova era do design de interação com a complementação das interfaces gráficas do usuário nas telas clássicas de computadores desktop pelas novas tecnologias que fazem uso de todo o corpo humano, de sensores e de novos dispositivos de entrada. De acordo com a 29 Pesquisa Anual de Administração e Uso de Tecnologia da Informação nas Empresas, realizada pela Fundação Getúlio Vargas de São Paulo (FGV-SP) e divulgada em abril de 2018, o Brasil conta com 220 milhões de smartphones ativos, superando a taxa de um aparelho por pessoa. Ao observar a familiarização tecnológica da população com dispositivos móveis, compreende-se que os smartphones e tablets são uma oportunidade para a acessibilidade digital. Embora não tenham sido inicialmente concebidos como tecnologias assistivas, estes dispositivos são altamente adaptáveis à função pelas configurações de interface, pelo fácil acesso à população e pela variabilidade de conteúdos e atividades disponíveis, podendo servir como valioso instrumento de inclusão digital para pessoas com deficiência.

Para o público com PC, também observa-se a oportunidade de uso e desenvolvimento destes aparatos, principalmente considerando o design de interação, por atuarem como estímulo para as funções motoras ao trabalharem com gestos e movimentos específicos. Bassani et al. (2014) definem que o Design de Interação "é uma área de pesquisa caracterizada pela interconexão de diferentes campos interdisciplinares, como interação humano-computador (IHC), ergonomia cognitiva, trabalho cooperativo apoiado por computador, entre outras". Desta forma, na perspectiva do design de interação e do design universal, as dificuldades enfrentadas por este público no uso destes dispositivos, considerando a complexidade para a execução de movimentos manuais precisos, devem ser analisadas e amenizadas através de projetos gráficos que levem em consideração as suas particularidades de interação.

Este estudo é parte de uma pesquisa exploratória focada em mapear possibilidades de aproximação do campo do design de interação e das tecnologias assistivas para o público com paralisia cerebral através de uma parceria do grupo de pesquisa do qual os autores fazem parte na Universidade do Estado de Santa Catarina (UDESC) com o Centro Catarinense de Reabilitação (CCR), localizado em Florianópolis/SC, que, com uma equipe multidisciplinar, atua na reabilitação adulto e infantil de diversas disfunções físicas, incluindo a paralisia cerebral. A pesquisa volta-se ao uso de novas tecnologias, entre elas os dispositivos touchscreen, como meio de acessibilidade e inclusão digital para o público com PC. Busca-se, também, contribuir para a movimentação fina deste público e, consequentemente, auxiliar nas terapias de reabilitação.

Desta forma, o trabalho objetivou, através de uma revisão bibliográfica sistemática, investigar e identificar estudos que abordassem como temática o uso de dispositivos touchscreen como tecnologia assistiva voltada às disfunções motoras finas e, mais especificamente, às condições da paralisia cerebral, de modo a identificar os caminhos de pesquisa nos 


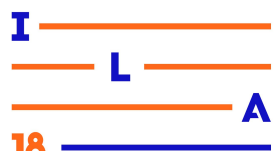

quais esta área tem se desenvolvido nos últimos anos, buscando observar seus avanços.

$\mathrm{O}$ artigo divide-se em quatro etapas principais, iniciando com uma breve contextualização teórica, de modo a expor a temática abordada. Em sequência, apresenta-se o método utilizado que, no caso, consiste em uma revisão bibliográfica sistemática, com sua string de busca e resultados quantitativos. Após esta apresentação, passa-se para os resultados obtidos através da leitura dos artigos selecionados, com informações acerca dos procedimentos utilizados e das conclusões. Por fim, os autores ressaltam pontos relevantes e trazem suas considerações finais, buscando explicitar as contribuições para trabalhos futuros a partir desta revisão.

\section{Método}

Para esta pesquisa, empregou-se como procedimento metodológico uma revisão bibliográfica sistemática (RBS) realizada nas bases de dados ProQuest, Scopus e Web of Science durante os meses de Abril e Maio de 2018. A revisão, focada na interação de pessoas com paralisia cerebral em dispositivos touchscreen e as possibilidades destes aparatos como tecnologia assistiva, utilizou a string de busca ("cerebral palsy") AND ("touchscreen" OR ("touch screen").

A primeira pesquisa, realizada no dia 26 de abril de 2018, resultou em 71 no artigos únicos, sendo 27 na ProQuest, 38 na Scopus e 6 na Web of Science. Logo de início, observou-se o baixo resultado quantitativo da string, o que demonstra a escassez de estudos com esta temática específica.

Pretendia-se, também, trabalhar com termos referentes ao design e à interação, porém os resultados se restringiam ainda mais quantitativamente e temia-se a perda de pesquisas relevantes com estas alterações na string. A tabela a seguir (Tabela 1) apresenta a string com seus filtros específicos aplicados em cada base.

\begin{tabular}{|l|l|}
\hline ProQuest & $\begin{array}{l}\text { ( "cerebral palsy" ) AND ( "touchscreen" or } \\
\text { "touch screen" ) } \\
\text { Filtros aplicados: Texto completo, Revisado por } \\
\text { especialistas, Periódicos acadêmicos, } \\
2014-2018\end{array}$ \\
\hline
\end{tabular}

\begin{tabular}{|c|c|}
\hline Scopus & $\begin{array}{l}\text { ALL ( ( "cerebral palsy" ) AND ( "touchscreen" } \\
\text { OR "touch screen" )) AND ( LIMIT-TO ( } \\
\text { PUBYEAR , 2018 ) OR LIMIT-TO ( PUBYEAR } \\
\text {, } 2017 \text { ) OR LIMIT-TO ( PUBYEAR , } 2016 \text { ) } \\
\text { OR LIMIT-TO ( PUBYEAR, 2015 ) OR } \\
\text { LIMIT-TO ( PUBYEAR, 2014 ) ) AND ( } \\
\text { LIMIT-TO ( DOCTYPE, "ar" ) ) AND ( } \\
\text { LIMIT-TO ( LANGUAGE, "English" ) OR } \\
\text { LIMIT-TO ( LANGUAGE, "Portuguese" ) ) }\end{array}$ \\
\hline $\begin{array}{l}\text { Web of } \\
\text { Science }\end{array}$ & $\begin{array}{l}\text { Tópico:(("cerebral palsy") AND ("touchscreen" or } \\
\text { "touch screen")) } \\
\text { Refinado por: Anos da publicação: ( } 2018 \text { OR } \\
2016 \text { OR } 2015 \text { OR } 2014 \text { ) } \\
\text { Tempo estipulado: Todos os anos.Índices: } \\
\text { SCI-EXPANDED, SSCI, A\&HCI, CPCI-S, } \\
\text { CPCI-SSH, ESCI. }\end{array}$ \\
\hline
\end{tabular}

Tabela 1. String de busca com respectivos filtros e bases de dados.

Fonte: Elaborado pelos autores, 2018.

Para a seleção de artigos, foram definidos alguns critérios de inclusão comuns às três bases utilizadas:

- Determinação da string com as palavras-chaves no idioma inglês;

- Seleção de artigos de periódicos lançados nos últimos cinco anos (de 2014 a 2018)

- Artigos redigidos em língua inglesa ou portuguesa

- Conter pesquisa relacionada a indivíduos com paralisia cerebral ou disfunções motoras que se assemelham aos casos de PC

- Apresentar informações sobre a interação, uso ou aplicativos de dispositivos touchscreen.

Após a investigação inicial, os autores aplicaram três filtros que, considerando os critérios apresentados anteriormente, visaram retirar os artigos que encontravam-se fora do contexto desta pesquisa. O primeiro consistiu na leitura dos títulos dos artigos, o segundo na leitura dos resumos e o terceiro, além da leitura diagonal (introdução, conclusão e métodos), excluiu aqueles sem acesso aos artigos completos, com acesso pago não disponível à instituição de origem dos autores e que não disponibilizavam o conteúdo completo nas bases de dados, consistindo apenas de resumos. As etapas da triagem e seus resultados quantitativos podem ser observados na tabela 2 . 


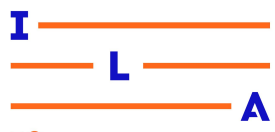

\begin{tabular}{|c|c|c|c|}
\hline BASE I & $\begin{array}{c}\text { Filtro 01 } \\
\text { (leitura } \\
\text { dos } \\
\text { títulos) }\end{array}$ & $\begin{array}{c}\text { Filtro 02 } \\
\text { (leitura dos } \\
\text { resumos) }\end{array}$ & $\begin{array}{c}\text { Filtro 03 (leitura } \\
\text { diagonal e } \\
\text { exclusão dos } \\
\text { não-gratuitos) }\end{array}$ \\
\hline ProQuest & 15 & 6 & 4 \\
\hline Scopus & 22 & 10 & 6 \\
\hline $\begin{array}{l}\text { Web of } \\
\text { Science }\end{array}$ & 5 & 5 & 2 \\
\hline
\end{tabular}

Tabela 2. Bases de dados, filtros e respectivos resultados quantitativos.

Fonte: Elaborado pelos autores, 2018.

O primeiro filtro obteve um total de 42 artigos, excluindo aproximadamente metade dos artigos da seleção inicial. Nesta etapa, foi possível perceber que muitos estudos excluídos abordavam somente uma das palavras-chave, dissertando sobre a paralisia cerebral ou sobre dispositivos touchscreen isoladamente. O segundo filtro resultou em 21 artigos e o terceiro, em 12. Retirando-se as duplicatas entre as bases, chegou-se no resultado final de 9 artigos para análise, expostos na tabela 3 . Em todas as etapas de filtragem, a base Scopus apresentou os maiores resultados quantitativos, demonstrando a concentração de pesquisas. A $W e b$ of Science apresentou poucos resultados, embora os 2 artigos utilizados na avaliação final sejam pertinentes a esta revisão.

\begin{tabular}{|c|c|c|c|c|}
\hline & Título & Autores & Periódico & Ano \\
\hline 1 & $\begin{array}{l}\text { User modeling for } \\
\text { people with } \\
\text { special needs }\end{array}$ & $\begin{array}{l}\text { Kurschl, } \\
\text { W.; } \\
\text { Augstein, } \\
\text { M.; } \\
\text { Burger, T.; } \\
\text { Pointner, } \\
\text { C. }\end{array}$ & $\begin{array}{l}\text { International } \\
\text { Journal of } \\
\text { Pervasive } \\
\text { Computing } \\
\text { and } \\
\text { Communicati } \\
\text { ons }\end{array}$ & 2014 \\
\hline 2 & $\begin{array}{l}\text { Speed-Accuracy } \\
\text { Testing on the } \\
\text { Apple iPad (R) } \\
\text { Provides a } \\
\text { Quantitative Test } \\
\text { of Upper Extremity } \\
\text { Motor } \\
\text { Performance in } \\
\text { Children with } \\
\text { Dystonia }\end{array}$ & $\begin{array}{l}\text { Bertucco, } \\
\text { M.; } \\
\text { Sanger, T. } \\
\text { D. }\end{array}$ & $\begin{array}{l}\text { Journal of } \\
\text { Child } \\
\text { Neurology }\end{array}$ & 2014 \\
\hline 3 & $\begin{array}{l}\text { Assessing mouse } \\
\text { alternatives to } \\
\text { access to } \\
\text { computer: A case }\end{array}$ & $\begin{array}{l}\text { Pousada, } \\
\text { T.; } \\
\text { Pareira, J.; } \\
\text { Groba, B.; } \\
\text { et al. }\end{array}$ & $\begin{array}{l}\text { Assistive } \\
\text { Technology }\end{array}$ & 2014 \\
\hline
\end{tabular}

\begin{tabular}{|c|c|c|c|c|}
\hline & $\begin{array}{l}\text { study of a user } \\
\text { with cerebral palsy }\end{array}$ & & & \\
\hline 4 & $\begin{array}{l}\text { Eye/Head } \\
\text { Tracking } \\
\text { Technology to } \\
\text { Improve HCl with } \\
\text { iPad Applications }\end{array}$ & $\begin{array}{l}\text { Lopez-Bas } \\
\text { terretxea, } \\
\text { A.; } \\
\text { Mendez-Z } \\
\text { orrilla, A.; } \\
\text { Garcia-Za } \\
\text { pirain, B. }\end{array}$ & Sensors & 2015 \\
\hline 5 & $\begin{array}{l}\text { Hand Function } \\
\text { With Touch } \\
\text { Screen } \\
\text { Technology in } \\
\text { Children With } \\
\text { Normal Hand } \\
\text { Formation, } \\
\text { Congenital } \\
\text { Differences, and } \\
\text { Neuromuscular } \\
\text { Disease }\end{array}$ & $\begin{array}{l}\text { Shin, D. } \\
\text { H.; Bohn, } \\
\text { D. K.; } \\
\text { Agel, J.; et } \\
\text { al. }\end{array}$ & $\begin{array}{l}\text { Journal of } \\
\text { Hand Surgery }\end{array}$ & 2015 \\
\hline 6 & $\begin{array}{l}\text { Increasing } \\
\text { participation in the } \\
\text { information society } \\
\text { by people with } \\
\text { disabilities and } \\
\text { their families in } \\
\text { lower-income } \\
\text { countries using } \\
\text { mainstream } \\
\text { technologies }\end{array}$ & $\begin{array}{l}\text { Barlott, T.; } \\
\text { Adams, } \\
\text { K.; Cook, } \\
\text { A. }\end{array}$ & $\begin{array}{l}\text { Universal } \\
\text { Access in the } \\
\text { Information } \\
\text { Society }\end{array}$ & 2016 \\
\hline 7 & $\begin{array}{l}\text { Effects of } \\
\text { activation method } \\
\text { and mental effort } \\
\text { on touchscreen } \\
\text { task performance } \\
\text { for users with and } \\
\text { without upper } \\
\text { extremity motor } \\
\text { control disabilities }\end{array}$ & $\begin{array}{l}\text { Chourasia, } \\
\text { A.; Sesto, } \\
\text { M.; Kuehn, } \\
\text { K.; et al. }\end{array}$ & $\begin{array}{l}\text { Universal } \\
\text { Access in the } \\
\text { Information } \\
\text { Society }\end{array}$ & 2017 \\
\hline 8 & $\begin{array}{l}\text { A methodological } \\
\text { approach for } \\
\text { designing and } \\
\text { developing } \\
\text { web-based } \\
\text { inventories of } \\
\text { mobile Assistive } \\
\text { Technology } \\
\text { applications }\end{array}$ & $\begin{array}{l}\text { Kouroupet } \\
\text { roglou, G.; } \\
\text { Pino, A.; } \\
\text { Riga, P. }\end{array}$ & $\begin{array}{l}\text { Multimedia } \\
\text { Tools and } \\
\text { Applications }\end{array}$ & 2017 \\
\hline 9 & $\begin{array}{l}\text { Use of iPads as } \\
\text { Assistive } \\
\text { Technology for } \\
\text { Students with } \\
\text { Disabilities }\end{array}$ & Ok, M. W. & TechTrends & 2018 \\
\hline
\end{tabular}

Tabela 3. Dados dos artigos finais da seleção. Fonte: Elaborado pelos autores, 2018.

É relevante, ainda, observar os locais dos quais os estudos são provenientes, de modo a avaliar a possibilidade de desenvolvendo de novos estudos 


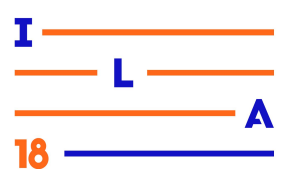

e identificar as referências nesta área de pesquisa atualmente. $O$ gráfico contido na imagem 1 exibe a localização dos autores dos artigos selecionados, demonstrando a concentração de pesquisadores nos Estados Unidos, seguido pela Espanha. Nota-se a falta de pesquisas com esta temática na América do Sul e, mais especificamente, no Brasil, o que destaca a relevância de desenvolver o campo da interação em telas sensíveis ao toque focado nos indivíduos com paralisia cerebral inseridos nesta região.

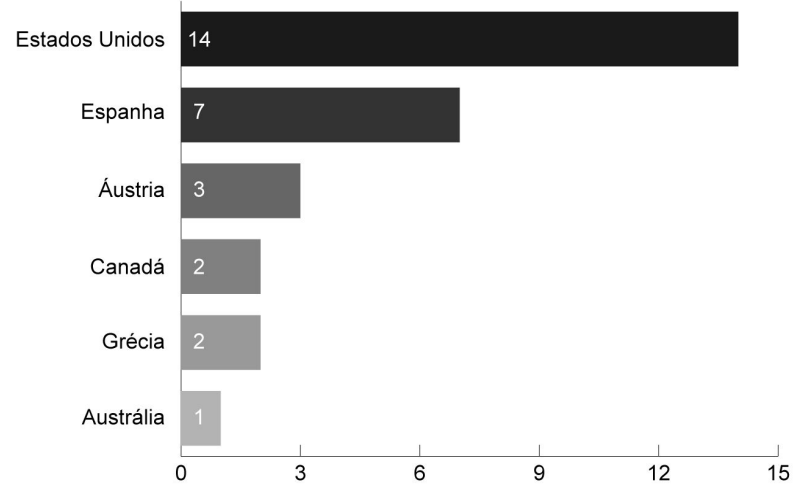

Imagem 1. Gráfico da localização de autores dos artigos selecionados.

Fonte: Elaborado pelos autores, 2018.

\section{Resultado}

Apresenta-se, a seguir, as principais informações coletadas na análise dos artigos selecionados, de modo a explicitar suas contribuições relevantes para esta pesquisa. Os artigos estão ordenados de acordo com sua temática principal, de modo a agrupar as contribuições semelhantes e facilitar o percurso de leitura. As temáticas se agrupam em questões acerca das interfaces, tipos de interação nos dispositivos (touch, touchless, eye e head-tracking), testes com dispositivos touchscreen e TAs alternativas e, ao final, aplicativos.

Barlott, Adams e Cook (2016) realizaram um estudo de caso em que celulares foram usados por pessoas com deficiências e seus cuidadores para acessar informações em uma comunidade com recursos limitados em Bogotá, na Colômbia. A fundamentação utilizada pelos autores no artigo foi considerada relevante para a pesquisa aqui apresentada. Eles investigam a possibilidade de tecnologias da informação e comunicação (ICTs) atuarem como tecnologias assistivas (TAs) para pessoas com deficiência em países subdesenvolvidos. Segundo dados da International Telecommunications Union (2011), 89\% das pessoas em países de baixa renda têm uma assinatura de telefonia móvel. Os autores observam que países de baixa renda tendem a fazer saltos tecnológicos, estando menos enraizados em tecnologias intermediárias (como, por exemplo, computadores pessoais e Internet de linha fixa), tendo facilidade na transição para novas tecnologias (como os celulares), do que os países de renda mais alta. Estes novos dispositivos possibilitam comunicações avançadas a um preço mais baixo. Os autores ressaltam, também, que a interface do computador está mudando de uma interação baseada no mouse, na qual utiliza-se um mouse e cursor para selecionar símbolos, para métodos mais diretos, como interfaces touchscreen e gestos. A denominação GUI (graphical user interface) é aplicada em interações que fazem uso de símbolos em tela (por exemplo, pastas ou ícones), que são selecionados utilizando um mouse, enquanto uma interface que depende de interação direta, como toque, movimento ou fala, é considerada uma interface natural do usuário (NUI). Observa-se um desafio no campo das tecnologias assistivas para essa adaptação de GUIs para NUIs, uma vez que a maioria das TAs atuais foram desenvolvidas a partir de interfaces gráficas do usuário. Nota-se, também, as vantagens de telefones smartphones e tablets para métodos de comunicação por serem mais acessíveis, prontamente disponíveis, possuírem redes confiáveis e requisitos de energia razoáveis. Desta forma, dispositivos móveis tradicionais estão sendo cada vez mais usados como tecnologias assistivas, uma vez que são mais acessíveis do que dispositivos específicos ou computadores.

Kurschl et al (2014) reportam diversos métodos e modelos para o desenvolvimento de interfaces de dispositivos como tablets e smartphones acessíveis a pessoas com deficiência motora e cognitiva, com foco no público infantil. Eles observam o crescimento das tendências touch e touchless nas modalidades de interação. Os tablets, por exemplo, estão cada vez mais difundidos e desempenham um papel crucial também para pessoas com deficiências, uma vez que são relativamente baratos, em comparação com 
hardwares específicos para pessoas com deficiências, oferecem novos métodos de entrada, são portáteis e fáceis de manusear. Em contrapartida, o touchless se torna relevante por permitir interação com o sistema por meio de gestos baseados em movimento parcial ou total do corpo, sendo útil a pessoas com deficiências motoras severas. Um desafio enfrentado é a alta variabilidade requerida, que pode ser parcialmente superada pela oferta de um alto nível de configurabilidade nas interfaces, mas demandando esforço para os desenvolvedores. Para tanto, o sistema deve ser continuamente atualizado sobre as habilidades, os handicaps e o progresso do indivíduo, a serem organizados em um modelo de usuário. $\mathrm{O}$ artigo busca analisar e desenvolver, através de testes com usuários, um modelo de usuário que poderia servir como base para $o$ comportamento adaptativo, de modo a reduzir os esforços de configuração. Um modelo de usuário organiza as habilidades, os handicaps e o progresso do usuário, podendo ser utilizado por desenvolvedores para configurar aplicativos para fornecer uma interface de usuário facilmente adaptável às capacidades individuais.

A pesquisa desenvolvida por Pousada et al (2014) buscou descrever o processo de avaliação de três dispositivos assistivos (touchscreen, joystick $\mathrm{e}$ trackball) para atender às necessidades de uma mulher com paralisia cerebral (nível III da escala MACS), a fim de fornecer acesso e uso do computador. Os dispositivos foram avaliados através de uma abordagem em três etapas: uso de um questionário para pré-selecionar potenciais tecnologias assistivas, uso da ferramenta eTAO para determinar a eficácia de cada um deles e uma entrevista semiestruturada para obter dados qualitativos. O melhor dispositivo que atendia às necessidades e prioridades do usuário foi o joystick. É importante considerar as necessidades e prioridades especiais dos usuários e experimentar dispositivos diferentes ao realizar um processo de seleção, pois a escolha pode variar com cada caso. Quanto aos dispositivos touchscreen, nota-se que este tipo é altamente intuitivo para selecionar diretamente os ícones mostrados na tela. No entanto, durante a execução de tarefas, observou-se que a operação desse dispositivo resultou em cansaço e fadiga da usuária, que apresentou dificuldades em completar as tarefas de arrastar e de alta precisão, principalmente ao realizar as tarefas de clique e clique duplo. Os observadores notaram que o posicionamento da usuária na cadeira de rodas não era ideal durante essa avaliação, fazendo com que ela adotasse posturas compensatórias. Com relação às percepções pessoais, a usuária não considerou a tela sensível ao toque um dispositivo eficaz para sua especificidade, embora acredite que poderia ser para outros usuários.

\section{Lopez-Basterretxea, Mendez-Zorrilla e} Garcia-Zapirain (2015) apresentam uma forma alternativa de interação nos dispositivos $i \mathrm{Pad}$, que faz uso da tecnologia de câmera frontal e de rastreamento dos olhos e da cabeça, buscando melhorar a interação homem-computador para pessoas com necessidades especiais. O processo de eye-tracking consiste em medir o foco de atenção (olhar) ou o movimento dos olhos em relação à cabeça. O principal problema com atuais sistemas de eye-tracking é a limitação de dispositivos disponíveis no mercado, tendo preços exorbitantes e sendo, portanto, em muitos casos, inacessíveis para usuários necessitados. No projeto desenvolvido pelos autores, o eye-tracking é integrado ao próprio sistema somente com configurações entre hardware (iPad) e software (iOS 7), sem a necessidade de um aparelho adicional específico. Após testes com 22 indivíduos, observou-se que o uso de óculos por parte dos usuários não interfere na detecção; olhos de cores claras (principalmente o verde) foram melhor detectados; a detecção das faces foi efetiva em todos os casos; a inclinação e altura do iPad provaram ser determinantes para o funcionamento do eye-tracking; e a iluminação adequada deve ser aplicada para melhores resultados.

Bertucco e Sange (2014) avaliaram o uso de telas de alta qualidade (um $\mathrm{iPad}$ ) de acordo com a lei de Fitts, que, matematicamente, afirma que o tempo de movimento para atingir um alvo aumenta com a distância e diminui à medida que o tamanho do alvo aumenta. No estudo, duas hipóteses foram testadas: de que a lei de Fitts se aplica a alvos em tablets touchscreen, em crianças típicas e em crianças com disfunções motoras e que o Índice de Desempenho de Fitts pode avaliar e comparar as habilidades sensoriais-motoras entre crianças com distonia, contrações musculares involuntárias que 
causam movimentos repetitivos ou torção, presente em diversos casos de paralisia cerebral. Para tanto, um experimento usando um software personalizado no iPad foi projetado para testar o paradigma da lei de Fitts em adultos saudáveis, crianças saudáveis e crianças com distonia. Os resultados fornecem evidências para a utilidade e validade do uso da lei de Fitts como ferramenta de diagnóstico quantitativo em crianças com distonia. Observou-se que testes em tablets com tela sensível ao toque podem ajudar a orientar o design de interfaces de usuário para maximizar a taxa de comunicação para crianças que dependem de dispositivos de comunicação aumentativa, por exemplo.

Shin et al (2015) elaboraram uma pesquisa para medir as movimentações manuais de 201 crianças com e sem deficiências em telas touchscreen. Embora os testes não tenham sido realizados somente em crianças com paralisia cerebral, observa-se a aplicabilidade deste método para as mesmas. Quatro tarefas foram medidas através da observação do investigador: toque em pontos em um grid $3 \times 4$, arrastar formas, uso da câmera e digitação de uma linha de texto. $\mathrm{O}$ teste leva de 60 a 120 segundos, no qual cada tarefa foi cronometrada de forma independente e o tempo total gravado. $\mathrm{O}$ estudo mostrou que crianças sem deficiência com idade inferior a 5 anos não apresentaram diferenças em relação às com deficiência e mesma idade, podendo indicar que nesta idade as habilidades motoras finas não se desenvolveram suficientemente para realizar a distinção. Indica-se, portanto, realizar testes com idades superiores. Ao final, estruturou-se o teste Minnesota Hand Function, que permite ao investigador mensurar a função manual de usuários em dispositivos touchscreen em menos de 5 minutos.

Chourasia et al (2017) afirmam que os métodos de ativação da tela sensível ao toque podem ajudar a resolver as dificuldades enfrentadas por pessoas com disfunções motoras, porém podendo apresentar compensações, como mais tempo para completar a tarefa. Os autores citam quatro métodos: tapping, crossing, take-off $\mathrm{e}$ swabbing. O estudo avaliou os métodos de acionamento tapping e take-off com participantes com e sem disfunções dos membros superiores ao completarem uma tarefa experimental para selecionar quatro itens de um menu em um tablet Samsung Galaxy Tab 10.1. Concluiu-se que para aplicações de seleção de menu de tablets touchscreen para indivíduos com e sem deficiências de controle motor da extremidade superior, o método take-off é mais preciso do que o tapping. No entanto, prefere-se o tapping por apresentar menos erros e menos tempo de conclusão da tarefa. Interfaces idênticas podem ser usadas para indivíduos com e sem incapacidades, embora tempo superior para a conclusão de tarefas deva ser considerado para indivíduos com deficiências. Os autores ressaltam que pesquisas futuras devem considerar o desenvolvimento e a avaliação de interfaces personalizadas para indivíduos com deficiências e a preferência por diferentes abordagens de ativação.

O estudo realizado por Kouroupetroglou, Pino e Riga (2017) relatou que as app stores (de sistemas operacionais como Android e iOS) não incluem uma categoria para tecnologias assistivas (TA) ou uma classificação por deficiência, dificultando o processo de pesquisa de aplicativos móveis que atendem às necessidades específicas do usuário com deficiência. A pesquisa objetivou propor uma nova abordagem metodológica para o design e desenvolvimento de inventários de aplicativos de TA. Embora não aborde o público com paralisia cerebral especificamente, é relevante para este artigo por abordar o agrupamento e busca de aplicativos voltados às diversas deficiências e, portanto, facilitando a interação digital de pessoas com PC. Para tanto, os autores apresentam os grupos de usuários de tecnologias assistivas, que consistem em pessoas com deficiência motora, visual, cognitiva, de fala e auditiva. A principal necessidade dos deficientes motores, especialmente para aqueles com comprometimento dos membros superiores, foco desta pesquisa, é conduzir as principais tarefas para operar a interface visual e de toque nos dispositivos móveis: segurar o dispositivo, ver a tela e tocá-la. Para tanto, os membros superiores precisam agir em conjunto. Os dedos são usados para apontar, selecionar, digitar, rolar, ampliar, girar e executar todos os gestos que uma interface touchscreen suporta. A mão é usada para segurar o dispositivo na posição correta; o braço leva o dispositivo ao local correto para que o usuário 


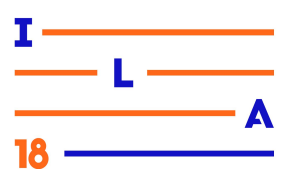

possa visualizá-lo e usá-lo. Aqueles com disfunções motoras que não conseguem realizar estes movimentos usam dispositivos especializados, como trackballs, sistemas de eye-tracking, rastreamento do movimento da cabeça, joysticks, touchpads e teclados modificados. Após esta fundamentação, alguns inventários existentes são apresentados, como o Special Needs Apps, BridgingsApps e o AssistIreland. Ao final, uma metodologia para o desenvolvimento de inventários de aplicativos de TA é explicada com suas seis etapas (pesquisa e localização de aplicativos móveis de TA; download e instalação dos aplicativos; teste e avaliação dos mesmos; criação de documentação consistente para cada aplicativo; projeto das instalações do inventário e sua construção; resultando no desenvolvimento e teste do inventário mATHENA.

Ok (2018) explora os potenciais benefícios do uso de $i$ Pads como tecnologia assistiva para apoiar as habilidades funcionais de estudantes com deficiências através de um estudo da literatura atual. Através da revisão realizada, 20 artigos foram analisados, sendo 14 deles, a maioria, focados em aplicativos de comunicação alternativa e aumentativa. Em outros estudos, o iPad foi usado para apoiar habilidades diárias (4), como compras online; vocacionais (1) e de lazer (1), utilizando o jogo Angry Birds para entretenimento. Os recursos multifuncionais do $\mathrm{iPad}$, com uma variedade de aplicativos funcionais para download e muitos recursos integrados, permitem que sirva como um dispositivo mais personalizado para atender as necessidades exclusivas de cada usuário.

Considerando as dificuldades daqueles com disfunções motoras, recursos integrados como AssistiveTouch e Switch Control permitem que os iPads sejam usados como uma tecnologia assistiva. $\mathrm{O}$ AssistiveTouch permite que os usuários com deficiências físicas que têm dificuldade em usar gestos de toque padrão criem gestos de toque personalizados. Por exemplo, se os usuários tiverem dificuldade para realizar o pinch com os dedos para aumentar ou diminuir o zoom da tela, poderão criar um gesto personalizado mais fácil para eles. Além disso, o Switch Control permite que os usuários usem vários switches adaptados ativados por Bluetooth para controlar o $i \mathrm{Pad}$, facilitando a interação. $\mathrm{O}$ artigo ainda apresenta exemplos de aplicativos e recursos on-line para encontrar esses aplicativos.

\section{Considerações finais}

O presente artigo apresentou, através de uma revisão bibliográfica sistemática, publicações que abordam a interação de pessoas com paralisia cerebral em dispositivos touchscreen, abrangendo, para a coleta de dados, estudos focados em outras disfunções motoras. Desta forma, foi possível concluir o objetivo inicial investigativo de identificar publicações com esta temática. A busca foi realizada em três bases de dados: na Scopus foram encontrados 6 artigos relevantes; na $\mathrm{Web}$ of Science, 2; e na ProQuest, 4. Retirando as duplicatas, foram analisados 9 artigos. Após uma investigação inicial, ordenou-se os artigos por data de publicação (tabela 3) e, posteriormente, nos resultados e discussões, agrupou-se de acordo com a temática abordada, buscando criar uma conexão entre eles. A análise exibiu artigos sobre as interfaces e tipos de interação nos dispositivos (touch, touchless, eye e head-tracking), testes e aplicativos, nesta ordem.

Entre as informações encontradas, destaca-se a facilidade de acesso a dispositivos touchscreen móveis em relação a outras TAs específicas ou computadores, seja pela possibilidade monetária ou pela portabilidade. Observa-se, também, a distinção entre GUI (graphical user interface), comum em sistemas desktop que necessitam de recursos externos como mouse ou teclado, e NUI (natural user interface), em crescente uso e desenvolvimento atual, que parte da interação humana direta para o acionamento dos dispositivos, desde a fala até o toque. As tecnologias assistivas enfrentam um desafio com esta transição, uma vez que a maioria das TAs foram desenvolvidas a partir de interfaces gráficas do usuário (BARLOTT, ADAMS e COOK, 2016). A passagem para as interfaces naturais do usuário (NUI) também está atrelada ao aumento contínuo de dispositivos móveis touchscreen à população.

Além do toque, outras interações se mostram possíveis com esse tipo de dispositivo, sendo o touchless e o eye-tracking campos a explorar, principalmente nos casos em que há 


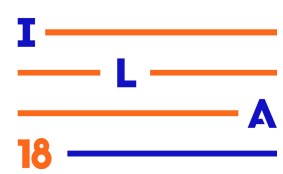

dificuldade do controle manual para a realização de gestos e movimentos finos. Diversos artigos ressaltaram a importância da customização ou adaptabilidade das interfaces ao usuário, como é o caso dos dispositivos $i \mathrm{Pads}$, tendo essas funcionalidades previstas em um modelo do usuário, por exemplo.

Observa-se, também, a importância de avaliar as necessidades dos usuários e a severidade da deficiência motora do indivíduo ao escolher o input digital adequado, uma vez que formas alternativas aos dispositivos touchscreen, como os joysticks, por exemplo, podem ser mais aconselháveis em casos específicos de disfunções motoras severas, conforme demonstrado pelo estudo realizado por Pousada et al (2014).

É necessário, ainda, desenvolver testes e formas de mensurar as capacidades individuais de manuseio e interação nestes dispositivos, considerando as classificações e os níveis de acometimento motor do público com paralisia cerebral. Mesmo com as classificações motoras atuais, percebe-se que o GMFCS e a MACS não avaliam as funções manuais finas, focando principalmente nas questões posturais, de marcha e do manuseio de objetos cotidianos pelos membros superiores em geral, restando uma lacuna para os gestos atrelados a dispositivos digitais, como os touchscreen, a ser estudada. Shin et al (2015) concluem que crianças abaixo de 5 anos podem não ter desenvolvido suas habilidades motoras finas de forma suficiente e, portanto, propõe-se que testes para estas mensurações sejam realizados acima dessa idade.

Foi possível notar que a maioria das pesquisas analisadas que realizaram testes com usuários utilizaram tablets ao invés de smartphones, possivelmente pelo tamanho de tela. Poucos estudos focaram a usabilidade exclusiva de pessoas com paralisia cerebral, uma vez que grande parte dos trabalhos foram realizados com disfunções motoras em geral, reforçando a possibilidade de estudos específicos para o público com paralisia cerebral e a relevância para a sua inclusão.

Observa-se, ainda, a necessidade de explorar os movimentos finos e gestos manuais possíveis pelo público com paralisia cerebral, de forma a orientar a concepção de interfaces adequadas às capacidades destes indivíduos. O uso das classificações expostas de maneira introdutória e utilizada por alguns dos pesquisadores aqui apresentados, como a GMFCS e a MACS, pode contribuir para a categorização destes movimentos possíveis.

Apoiado nas considerações anteriormente expostas, propõe-se, como sugestão para futuros estudos, realizar novos testes de interação com dispositivos touchscreen por pessoas com PC, de modo a reunir informações acerca da movimentação possível de acordo com os níveis de acometimento da paralisia cerebral e possibilitando elencar parâmetros para o projeto de interfaces digitais que facilitem a usabilidade destes aparelhos.

\section{Referências bibliográficas}

2010, CENSO IBGE. Censo IBGE 2010.

Disponível em:

$<$ http://www.ibge.gov.br/home/estatistica/populacao /censo2010/>. Acesso em: 10 abril 2018.

BARLOTT, T.; ADAMS, K.; COOK, A. Increasing participation in the information society by people with disabilities and their families in lower-income countries using mainstream technologies. Universal Access in the Information Society, Vol. 15, No 2, p. 189-198. 2016.

BASSANI, Patrícia B. Scherer et al. Usabilidade e acessibilidade no desenvolvimento de interfaces para ambientes de educação à distância. 2010 . Disponível em: $<\mathrm{http}: / /$ seer.ufrgs.br $>$. Acesso em: 10 set. 2017.

BERTUCCO, M.; SANGER, T. Speed-Accuracy Testing on the Apple iPad (R) Provides a Quantitative Test of Upper Extremity Motor Performance in Children with Dystonia. Journal of Child Neurology, Vol. 29, No 11, p. 1460-1466. 2014.

BRASIL. Ministério da Saúde. Secretaria de Atenção à Saúde. Departamento de Ações 


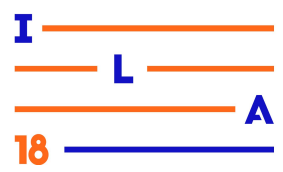

Programáticas Estratégicas. Diretrizes de atenção à pessoa com paralisia cerebral. Brasília: Ministério da Saúde, 2014. 72 p.

CHOURASIA, A., et al. Effects of activation method and mental effort on touchscreen task performance for users with and without upper extremity motor control disabilities. Universal Access in the Information Society, Vol. 16, No 2, p. 469-481. 2017.

CIASCA, S.M.; MOURA-RIBEIRO, M.V.L.; TABAQUIM, M.L.M. Aprendizagem e paralisia cerebral. In: ROTTA, N.T.; OHLWEILER, L.; RIESGO, R.S. Transtornos da Aprendizagem: abordagem neurobiológica e multidisciplinar. Porto Alegre: Artmed, 2006. p. 409-415.

\section{ELIASSON, Ann Christin et al. Manual Ability}

Classification System: Manual para crianças com paralisia cerebral 4-18 anos. 2010. Disponível em: $<$ www.macs.nu/files/MACS_Portuguese-Brazil_201 0.pdf $>$. Acesso em: 18 jun. 2018.

FGV. 29a Pesquisa Anual de Administração e Uso de Tecnologia da Informação nas Empresas. Disponível em:

https://eaesp.fgv.br/ensinoeconhecimento/centros/ci a/pesquisa. Acesso em: 18 jun. 2018.

KOUROUPETROGLOU, G.; PINO, A.; RIGA, P. $A$ methodological approach for designing and developing web-based inventories of mobile Assistive Technology applications. Multimedia Tools and Applications, Vol. 76, $\mathrm{N}^{\mathrm{o}}$ 4, p. 5347-5366. 2017.

KURSCHL, W., et al. User modeling for people with special needs. International Journal of Pervasive Computing and Communications, Vol. 10, No 3, p. 313-336. 2014.

LOPEZ-BASTERRETXEA, A.; MENDEZ-ZORRILLA, A.; GARCIA-ZAPIRAIN, $B$. Eye/Head Tracking Technology to Improve HCI with iPad Applications. Sensors, Vol. 15, $\mathrm{N}^{\circ}$ 2, p. 2244-2264. 2015.
OK, M. W. Use of iPads as Assistive Technology for Students with Disabilities. TechTrends, Vol. 62, $\mathrm{N}^{\mathrm{o}}$ 1, p. 95-102. 2018.

POUSADA, T., et al. Assessing mouse alternatives to access to computer: A case study of a user with cerebral palsy. Assistive Technology, Vol. 26, No 1, p. 33-44. 2014.

SCPE, Surveillance of Cerebral Palsy in Europe. Paralisia Cerebral. Disponível em:

$<$ http://www.scpenetwork.eu/pt/my-scpe/manual-de -referencia-e-formacao/pc-e-subtipos-de-pc/>. Acesso em: 18 jun. 2018.

SHIN, D., et al. Hand Function With Touch Screen Technology in Children With Normal Hand Formation, Congenital Differences, and Neuromuscular Disease. Journal of Hand Surgery, Vol. 40, No 5, p. 922-927. 2015.

\section{LOPEZ-BASTERRETXEA, A.;} MENDEZ-ZORRILLA, A.; GARCIA-ZAPIRAIN, $B$. Eye/Head Tracking Technology to Improve HCI with iPad Applications. Sensors, Vol. 15, $\mathrm{N}^{\circ}$ 2, p. 2244-2264. 2015.

OK, M. W. Use of iPads as Assistive Technology for Students with Disabilities. TechTrends, Vol. 62, $\mathrm{N}^{\mathrm{o}} 1$, p. 95-102. 2018.

POUSADA, T., et al. Assessing mouse alternatives to access to computer: A case study of a user with cerebral palsy. Assistive Technology, Vol. 26, No 1, p. 33-44. 2014.

SAFFER, D. Designing Gestural Interfaces: Touchscreens and Interactive Devices. Canada: O'reilly Media, 2009.

SCPE, Surveillance of Cerebral Palsy in Europe. Paralisia Cerebral. Disponível em:

$<$ http://www.scpenetwork.eu/pt/my-scpe/manual-de -referencia-e-formacao/pc-e-subtipos-de-pc/s. Acesso em: 8 set. 2017.

SHIN, D., et al. Hand Function With Touch Screen Technology in Children With Normal Hand Formation, Congenital Differences, and 


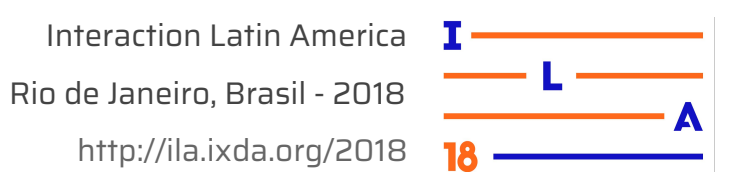

Neuromuscular Disease. Journal of Hand Surgery,

Vol. 40, No 5, p. 922-927. 2015. 